\title{
LUSTERNIK-SCHNIRELMANN CATEGORY AND COBORDISM
}

\author{
HARPREET SINGH
}

(Communicated by Haynes R. Miller)

\begin{abstract}
The Lusternik-Schnirelmann category of a manifold $M$ is the smallest integer $k$ that $M$ can be covered by $k$ open sets each of which is contractible in $M$. It is an upper bound for the length of nonzero products of Stiefel-Whitney classes of $M$. The object of this paper is to use this restriction, on the length of nonzero products, to investigate the cobordism classes of manifolds with category less than or equal to three.
\end{abstract}

Introduction. Let $M$ be an $n$-dimensional manifold. ${ }^{1}$ The Lusternik-Schnirelmann category of $M$, cat $(M)$, is the smallest integer $k$ such that $M$ can be covered by $k$ open sets each of which is contractible in $M$. The object of this paper is to identify the cobordism class of a manifold $M$ with cat $(M) \leq 3$.

Thom [11] has shown that the Stiefel-Whitney numbers (see [7]) of $M$ determine the cobordism class of $M$. It is also known (see [4]) that cat $(M)$ is greater than the length of the longest nonzero product in $H^{*}(M)$-the coefficient ring will always be $\mathbf{Z}_{2}$. In particular if cat $(M) \leq 3$, then the only possibly nonzero Stiefel-Whitney numbers are $W_{i} W_{n-i}[M]$ for $0 \leq i \leq n$. Mielke [5] has shown that if $n \equiv 3(\bmod 4)$, then all these numbers are zero and thus $M$ is a boundary. We will see in $\S 2$ that this is a special case of the following:

THEOREM 2.12'. If $M$ is an $n$-dimensional manifold with cat $(M) \leq 3$, then $M$ is either a boundary or else one of the following is true:

(1) $n=2^{s}, s \geq 1$, and $M$ is cobordant to $\left(\mathrm{R} P^{2}\right)^{2^{s-1}}$.

(2) $n=2^{r}\left(2^{s}+1\right), r \geq 0, s \geq 2$, and $M$ is cobordant to $(\tilde{M})^{2^{r}}$, where

$$
\tilde{M}=\bigcup_{j=0}^{2^{s-2}} P\left(1,2^{s-1}-2 j\right) \times N_{j},
$$

$N_{j}$ is the degree $4 j$ term in the formal inverse $\left(1+\mathrm{C} P^{2}+\mathbf{C} P^{4}+\mathrm{C} P^{6}+\cdots\right)^{-1}$, $P(1, m)$ is the Dold manifold $[\mathbf{3}]\left(S^{1} \times \mathbf{C} P^{m}\right) / \mathbf{Z}_{2}$, and $\bigcup$ denotes disjoint union.

In $\S 1$, we develop the machinery of Poincaré algebras and introduce a weaker concept, the cobordism category of a manifold, denoted cobcat $(M)$, which has the advantage of being a cobordism invariant. This simplifies the proofs of $\S 2$ considerably. In $\S 2$ we investigate the cobordism class of a manifold $M$ with cat $(M) \leq 3$, culminating with Theorem 2.12 .

Received by the editors June 16, 1986. Parts of this paper were presented at the October 1986 meeting of the A.M.S. in Charlotte, North Carolina.

1980 Mathematics Subject Classification (1985 Revision). Primary 57R75, 55M30.

${ }^{1}$ All manifolds are to be closed, smooth, and compact. 
This work forms a part of the author's dissertation at the University of Virginia. The author would like to thank Dr. R. E. Stong, for suggesting this problem, and for his guidance during the dissertation.

1. Poincaré algebras and cobcat $(M)$. Let $B O$ be the classifying space of the infinite orthogonal group. It is well known, (see [7]), that the mod 2 cohomology of $B O$ is a $\mathbf{Z}_{2}$-polynomial algebra on the Stiefel-Whitney classes $W_{i}, i=1,2, \ldots$ If $M$ is an $n$-dimensional manifold, then associated to $M$ is a homomorphism $\tau: H^{n}(B O) \rightarrow \mathbf{Z}_{2}$ defined by $\tau\left(W_{i_{1}} \cdots W_{i_{p}}\right)=W_{i_{1}} \cdots W_{i_{p}}[M]$ where $i_{1}+\cdots+i_{p}=n$ is a partition of $n$, and $W_{i_{1}} \cdots W_{i_{p}}[M]$ is the corresponding Stiefel-Whitney number of $M$. Let $J=\left\{x \in H^{*}(B O) \mid \operatorname{dim}(x)>n\right.$ or $\tau(x y)=0$ for all $\left.y \in H^{n \text {-dim(x) }}(B O)\right\}$. It is easy to see that $J$ is an ideal of the graded algebra $H^{*}(B O)$. Let $p^{*}$ denote the quotient algebra and let $f: H^{*}(B O) \rightarrow P^{*}$ be the natural quotient map. Then we have

PROPOSITION 1.1 [10]. (1) If $M$ is not a boundary, then $P^{*}$ is an n-dimensional graded algebra with Poincaré duality, and $M$ is a boundary iff $P^{*}=0$.

(2) The Steenrod algebra acts on $P^{*}$ with the action given by

$$
\mathrm{Sq}^{i}(f(x))=f\left(\mathrm{Sq}^{i}(x)\right) \text {. }
$$

(3) $f: H^{n}(B O) \rightarrow p^{n} \cong \mathbf{Z}_{2}$ is the homomorphism $\tau$, i.e., if $x \in H^{n}(B O)$, then $f(x)=0$ if and only if $\tau(x)=x[M]=0$.

(4) If $x \in J$, then $f(x)=0$.

$P^{*}$ will be called the Poincaré algebra associated to $M$. It is easy to see that the usual relations between the Stiefel-Whitney classes, the Wu classes, and the Steenrod squares still hold in $P^{*}$.

DEFinition. Let $A^{*}$ be any graded algebra. An element $x \in A^{*}$ will be called $k$-decomposable if it is zero or the sum of products $y_{1} \cdots y_{p}$ where $\operatorname{dim}\left(y_{i}\right)>0$ for all $i$ and $p \geq k$.

DEFINITION. Let $M$ be an $n$-dimensional manifold. The cobordism category of $M$, cobcat $(M)$, is the smallest integer $k$ such that $W_{i_{1}} \cdots W_{i_{p}}[M]=0$ for all partitions $i_{1}+\cdots+i_{p}=n$ with $k \leq p \leq n$. If no such $k$ exists, then define $\operatorname{cobcat}(M)=n+1$.

Clearly cobcat $(M)$ is a cobordism invariant and cobcat $(M) \leq \operatorname{cat}(M)$.

PROPOSITION 1.2. If $M$ is an $n$-dimensional manifold with cobcat $(M) \leq k$, and $P^{*}$ is the associated Poincaré algebra, then

(1) If $x \in P^{*}$ is $k$-decomposable, then $x$ is zero.

(2) If $x \in P^{*}$ is $(k-1)$-decomposable and $\operatorname{dim}(x)<n$, then $x$ is zero.

PROOF. Let $i_{1}+\cdots+i_{p} \leq n$ where $p \geq k$. Then for each $x \in H^{*}(B O)$ with $\operatorname{dim}(x)=n-\left(i_{1}+\cdots+i_{p}\right)$ we have $W_{i_{1}} \cdots W_{i_{p}} \cdot x[M]=0$, and hence, $W_{i_{1}} \cdots W_{i_{p}}=0$ in $P^{*}$. Clearly this is also true if $i_{1}+\cdots+i_{p}>n$. Thus every $k$-decomposable element in $H^{*}(B O)$ is zero in $P^{*}$. Since every $k$-decomposable element in $P^{*}$ is the image of a $k$-decomposable element in $H^{*}(B O)$, we have (1).

Let $x$ be a $(k-1)$-decomposable element of $H^{*}(B O)$ with $\operatorname{dim}(x)<n$. Then for all $y \in H^{n \text {-dim(x) }}(B O), x y$ is $k$-decomposable and so by the above it is zero in $P^{*}$. Thus $x$ is zero in $P^{*}$. 
Proposition 1.3. Let $M$ be an n-dimensional manifold, and let $c \in H^{1}(M)$. Let $N$ be the submanifold of $M$ dual to $c$. If $c^{2} x[M]=0$ for all $x$ in the subalgebra of $H^{*}(M)$ generated by $c, W_{1}, \ldots, W_{n}$ with $\operatorname{dim}(x)=n-2$, then $W_{i_{1}} \cdots W_{i_{p}}[N]=$ $c W_{i_{1}} \cdots W_{i_{p}}[M]$ for any partition $i_{1}+\cdots+i_{p}=n-1$.

Proof. It is known (see [9, p. 78]) that $W(N)=j^{*}(W(M)) /\left(1+j^{*}(c)\right)$ and $j^{*}(x)[N]=c x[M]$ for all $x \in H^{n-1}(M)$, where $j$ is the inclusion $N \subset M$. Thus

$$
W(N)=j^{*}\left(1+W_{1}+W_{2}+\cdots+W_{n}\right)\left(1+c+c^{2}+\cdots\right)
$$

and so $W_{i}(N)=j^{*}\left(W_{i}+c W_{i-1}+\cdots+c^{i}\right)$. Thus for any partition $i_{1}+\cdots+i_{p}=n-1$, we have

$$
\begin{aligned}
W_{i_{1}} \cdots W_{i_{p}}[N] & =j^{*}\left(\prod_{k=1}^{p}\left(W_{i_{k}}+\cdots+c^{i_{k}}\right)\right)[N] \\
& =c\left(\prod_{k=1}^{p}\left(W_{i_{k}}+\cdots+c^{i_{k}}\right)\right)[M] \\
& =c W_{i_{1}} \cdots W_{i_{p}}[M] \text { by the hypothesis on } c .
\end{aligned}
$$

2. Manifolds with cobcat $(M) \leq 3$. Throughout this section $M$ will denote an $n$-dimensional manifold with $\operatorname{cobcat}(M) \leq 3$ and with associated Poincaré algebra $P^{*}$.

From Proposition 1.2, we know that if an element $x \in P^{*}$ is 3-decomposable, or if $x$ is decomposable and $\operatorname{dim}(x)<n$, then $x$ is zero in $P^{*}$. Thus, in particular, we only need to look at the products $W_{i} W_{n-i}, i=0,1, \ldots, n$, in $P^{*}$. We may assume $i \leq n / 2$.

LEMMA 2.1. (1) If $x W_{i}=0$ in $P^{*}$, where $\operatorname{dim}(x)=n-i$ and $0<i<n$, then $x=0$ in $P^{*}$.

(2) For $j>0$,

$$
W_{2 j+1}= \begin{cases}\mathrm{Sq}^{1}\left(W_{2 j}\right), & \text { if } 2 j+1<n, \\ 0, & \text { if } 2 j+1=n .\end{cases}
$$

ProOF. (1) By hypothesis and the fact that $\operatorname{cobcat}(M) \leq 3$, we see that all numbers divisible by $x$ are zero, and so $x=0$ in $P^{*}$.

(2) Now, $\mathrm{Sq}^{1}\left(W_{2 j}\right)=W_{1} W_{2 j}+W_{2 j+1}$ in $P^{*}$. If $2 j+1<n$, then since $W_{1} W_{2 j}$ is decomposable, it is zero. If $2 j+1=n$, then $W_{1} W_{2 j}=\operatorname{Sq}^{1}\left(W_{2 j}\right)$.

LEMMA 2.2. If $n$ is even and $n>2$, then $W_{i}=0$ in $P^{*}$ for all odd $i$.

Proof. If $i=1$, then by Lemma $2.1, W_{n-1}=\operatorname{Sq}^{1}\left(W_{n-2}\right)$ and so $W_{1} W_{n-1}=$ $\mathrm{Sq}^{1}\left(W_{n-1}\right)=\operatorname{Sq}^{1}\left(\operatorname{Sq}^{1}\left(W_{n-2}\right)\right)=0$. If $i=2 j+1$, where $j>0$, then $n-i=2 k+1$ where $k>0$, and so by Lemma 2.1,

$$
W_{i} W_{n-i}=\operatorname{Sq}^{1}\left(W_{2 j}\right) \operatorname{Sq}^{1}\left(W_{2 k}\right)=\operatorname{Sq}^{1}\left(W_{2 j} \operatorname{Sq}^{1}\left(W_{2 k}\right)\right)=v_{1} W_{2 j} \operatorname{Sq}^{1}\left(W_{2 k}\right),
$$

which is 3-decomposable and hence zero. Thus by Lemma $2.1, W_{i}$ is zero in $P^{*}$ for all odd $i$.

PROPOSITION 2.3. If $M$ is a nonbounding $n$-dimensional manifold with

$$
\operatorname{cobcat}(M) \leq 3,
$$


where $n$ is even and $n>2$, then $M$ is cobordant to $N \times N$, where $N$ is also nonbounding and cobcat $(N) \leq 3$.

PrOOF. By Lemma 2.2, all Stiefel-Whitney numbers of $M$ divisible by $W_{i}$ for $i$ odd are zero and so by [6], $M$ is cobordant to $N \times N$. From [6] we also know that $W_{i_{1}} \cdots W_{i_{p}}[N]=W_{2 i_{1}} \cdots W_{2 i_{p}}[M]$ for any partition $i_{1}+\cdots+i_{p}=n / 2$. Thus, clearly $N$ is not a boundary and $\operatorname{cobcat}(N)=\operatorname{cobcat}(M) \leq 3$.

COROLlARY 2.4. Let $n=2^{t} m$ where either $m$ is odd and $m \geq 3$, or $m=2$. Then either $M$ is a boundary or else $M$ is corbordant to $(N)^{2^{t}}$, where $N$ is a nonbounding $m$-dimensional manifold with $\operatorname{cobcat}(N) \leq 3$.

PrOOF. Proceed inductively using Proposition 2.3.

COROLlARY 2.5. Let $M$ be an $n$-dimensional manifold with cobcat $(M) \leq 3$.

(1) If $n=2^{s}, s>0$, then either $M$ is a boundary or else $M$ is cobordant to $\left(\mathbf{R} P^{2}\right)^{2^{-1}}$.

(2) If $n=3\left(2^{s}\right), s \geq 0$, then $M$ is a boundary.

PROOF. By Corollary 2.4, all we need to do is to look at $\mathfrak{N}_{2}$ and $\mathfrak{N}_{3} \cdot \mathfrak{N}_{2} \cong \mathbf{Z}_{2}$ with generator $\mathbf{R} P^{2}$ and cobcat $\left(\mathbf{R} P^{2}\right) \leq 3$. Also $\mathfrak{N}_{3}=0$.

LEMMA 2.6. Let $n=2^{r}+m$, where $1 \leq m<2^{r}$. Then

(1) $W_{2^{s}+k}=\mathrm{Sq}^{k}\left(W_{2^{s}}\right)$ in $P^{*}$ if $0 \leq k<2^{s}$ and $2^{s}+k<n$.

(2) $W_{j}=0$ in $P^{*}$ if $0<j \leq m$.

ProOF. (1) $\mathrm{Sq}^{k}\left(W_{2^{s}}\right)=W_{2^{s}+k}+\sum_{t=0}^{k-1}\left(\begin{array}{c}2^{s}-k+t-1 \\ t\end{array}\right) W_{k-t} W_{2^{s}+t}$, and decomposable elements of dimension less than $n$ are zero in $\rho^{*}$.

(2) Let $0<j \leq m$. Then $n-j=2^{r}+k$ where $0 \leq k<2^{r}$ and $2^{r}+k<n$. Thus by (1), $W_{n-j}=\operatorname{Sq}^{k}\left(W_{2 r}\right)$. It is known that the Wu class $v_{j}$ is decomposable, and hence zero in $P^{*}$, if $j$ is not a power of 2 . Thus, $W_{2^{r}}=v_{2^{r}}$ in $P^{*}$ and since $2^{r}>n / 2$, we have $W_{2^{r}}=v_{2^{r}}=0$ in $P^{*}$. Thus $W_{n-j}=0$ in $P^{*}$ and so by Lemma $2.1, W_{j}=0$ in $P^{*}$.

Proposition 2.7. If $v_{1}=v_{2}=0$ in $P^{*}$ and if $n>3$ is odd, then $M$ is a boundary.

PROOF. let $2<2^{t} \leq 2^{r-1}$. Then

$$
v_{2^{t}} W_{n-2^{t}}=\mathrm{Sq}^{2 t} \operatorname{Sq}^{1}\left(W_{n-2^{t}-1}\right)
$$

by Lemma 2.1 , and by the Adem relations $\mathrm{Sq}^{2} \mathrm{Sq}^{2^{t}-1}=\mathrm{Sq}^{2^{t}+1}+\mathrm{Sq}^{2 t} \mathrm{Sq}^{1}=$ $\mathrm{Sq}^{1} \mathrm{Sq}^{2^{t}}+\mathrm{Sq}^{2^{t}} \mathrm{Sq}^{1}$. Thus

$$
v_{2^{t}} W_{n-2^{t}}=\mathrm{Sq}^{2} \mathrm{Sq}^{2^{t}-1}\left(W_{n-2^{t}-1}\right)+\mathrm{Sq}^{1} \mathrm{Sq}^{2^{t}}\left(W_{n-2^{t}-1}\right)=0
$$

in $P^{*}$ by hypothesis. Thus $v_{2^{t}}=0$ in $P^{*}$ for any $1 \leq 2^{t} \leq 2^{r-1}$ and so $v=1$ in $P^{*}$. Thus $M$ is a boundary.

Let $\alpha(n)$ denote the number of terms in the 2-adic expansion of $n$.

COROLlARY 2.8. If $M$ is an n-dimensional manifold with $\alpha(n) \geq 3$ and with $\operatorname{cobcat}(M) \leq 3$, then $M$ is a boundary.

PROOF. By Corollary 2.4 we may assume that $n$ is odd and write $n=2^{r}+m$ where $3 \leq m<2^{r}$ and $m$ is odd. By Lemma $2.6, W_{1}=W_{2}=0$ in $P^{*}$ and so by Proposition 2.7, $M$ is a boundary. 
COROllaRY 2.9. Let $M$ be an $n$-dimensional manifold with cobcat $(M) \leq 3$. If $n=2^{s}+1$ where $s \geq 2$, and $W_{2} W_{n-2}[M]=0$, then $M$ is a boundary.

ProOF. By Lemma 2.6, $W_{1}=0$ in $P^{*}$. If $W_{2} W_{n-2}[M]=0$, then $W_{2}=0$ in $P^{*}$ and so by Proposition 2.7, $M$ is a boundary.

PROPOSITION 2.10. Let $n=2^{s}+1$, where $s \geq 2$. If there exist two nonbounding $n$-dimensional manifolds $M$ and $M^{\prime}$ with cobcat $(M) \leq 3$ and cobcat $\left(M^{\prime}\right) \leq 3$, then they are cobordant. that

ProOF. By Corollary 2.9, $W_{2} W_{n-2}[M]=W_{2} W_{n-2}\left[M^{\prime}\right]=1$. It is well known

$$
W_{i_{1}} \cdots W_{i_{p}}\left[M \cup M^{\prime}\right]=W_{i_{1}} \cdots W_{i_{p}}[M]+W_{i_{1}} \cdots W_{i_{p}}\left[M^{\prime}\right]
$$

where $M \cup M^{\prime}$ is the disjoint union of $M$ and $M^{\prime}$. Thus clearly cobcat $\left(M \cup M^{\prime}\right) \leq 3$, and $W_{2} W_{n-2}\left[M \cup M^{\prime}\right]=0$, and so by Corollary $2.9, M \cup M^{\prime}$ is a boundary.

THEOREM 2.11. Let

$$
M=\bigcup_{j=0}^{2^{s-2}} P\left(1,2^{s-1}-2 j\right) \times N_{j}
$$

where $s \geq 2, P\left(1,2^{s-1}-2 j\right)$ is a Dold manifold, $N_{j}$ is the degree $4 j$ term in the formal inverse $\left(1+\mathbf{C} P^{2}+\mathbf{C} P^{4}+\mathbf{C} P^{6}+\cdots\right)^{-1}$, and $\bigcup$ denotes disjoint union. Then $M$ is nonbounding, and cobcat $(M) \leq 3$.

PROOF. Let $P_{j}$ denote $P\left(1,2^{s-1}-2 j\right)$. There is a characteristic number $S_{n}$ (see [7]) with the property that $S_{n}[L]=0$ if $L$ is a decomposable $n$-dimensional manifold. Thus $S_{2^{s}+1}[M]=S_{2^{s}+1}\left[P\left(1,2^{s-1}\right)\right]=1$. Thus $M$ is not a boundary. Let $i_{1}+\cdots+i_{p}=2^{s}+1$ be a partition of $2^{s}+1$. Then

$$
W_{i_{1}} \cdots W_{i_{p}}[M]=\sum_{j=0}^{2^{s-2}} W_{i_{1}} \cdots W_{i_{p}}\left[P_{j} \times N_{j}\right] .
$$

$P(1,0)$ is a boundary so we may assume $j<2^{s-2}$. It is known (see [3]) that $W\left(P_{j}\right)=(1+c)(1+c+d)^{2^{s-1}-2 j+1}$, where $\operatorname{dim}(c)=1, \operatorname{dim}(d)=2$, and $c^{2}=$ $d^{2^{o-1}-2 j+1}=0$. Thus

$$
\begin{aligned}
W\left(P_{j}\right) & =(1+c)(1+c+d)(1+c+d)^{2^{s-1}-2 j} \\
& =(1+d+c d)(1+d)^{2^{s-1}-2 j} .
\end{aligned}
$$

Clearly $W_{\text {odd }}\left(N_{j}\right)=0$, and so for $i>1, W_{2 i+1}\left(P_{j} \times N_{j}\right)=\bar{c} \bar{d} y$, where $y$ is the degree $(2 i-2)$ term in $(1+d)^{2^{s-1}-2 j} \times W\left(N_{j}\right), \bar{c}=c \times 1$ and $\bar{d}=d \times 1 . \bar{c}^{2}=0$, and so $W_{\text {odd }} W_{\text {odd }}\left(P_{j} \times N_{j}\right)=0$. Thus the only possibly nonzero Stiefel-Whitney number of $M$ are of the form

$$
W_{2 i+1} W_{2 i_{2}} \cdots W_{2 i_{p}}[M]=\sum_{j=0}^{2^{s-2}} \bar{c} \bar{d} y W_{2 i_{2}} \cdots W_{2 i_{p}}\left[P_{j} \times N_{j}\right]
$$

$c^{2}=0$, and so by Proposition 1.3 , there is a submanifold $\tilde{P}_{j}$ (of $P_{j}$ ) dual to 
c. It is easy to see that $\tilde{P}_{j}$ is cobordant to $\mathbf{C} P^{2^{s-1}-2 j}$ with $W\left(\mathbf{C} P^{2^{s-1}-2 j}\right)=$ $(1+d)^{2^{s-1}-2 j+1}$. By using the Whitney product formula repeatedly one can easily show that

$$
\bar{c} \bar{d} y W_{2 i_{2}} \cdots W_{2 i_{p}}\left[P_{j} \times N_{j}\right]=\bar{d} y W_{2 i_{2}} \cdots W_{2 i_{p}}\left[\mathbf{C} P^{2^{s-1}-2 j} \times N_{j}\right] .
$$

Furthermore, $y$ is the degree $2 i-2$ term in

$$
\begin{aligned}
(1+d)^{2^{s-1}-2 j} \times W\left(N_{j}\right) & =\frac{W\left(\mathbf{C} P^{2^{s-1}-2 j}\right)}{(1+d)} \times W\left(N_{j}\right) \\
& =\left(1+\bar{d}+\bar{d}^{2}+\cdots\right) W\left(\mathbf{C} P^{2^{s-1}-2 j} \times N_{j}\right) .
\end{aligned}
$$

Thus $y=\sum_{k=0}^{i-1} \bar{d}^{k} W_{2 i-2-2 k}\left(\mathbf{C} P^{2^{s-1}-2 j} \times N_{j}\right)$, and so

$$
\bar{c} \bar{d} y W_{2 i_{2}} \cdots W_{2 i_{p}}\left[P_{j} \times N_{j}\right]=\sum_{k=0}^{i-1} \bar{d}^{k+1} W_{2 i-2-2 k} W_{2 i_{2}} \cdots W_{2 i_{p}}\left[\mathbf{C} P^{2^{s-1}-2 j} \times N_{j}\right] .
$$

Hence

$$
W_{2 i+1} W_{2 i_{2}} \cdots W_{2 i_{p}}[M]=\sum_{k=0}^{i-1} \tilde{d}^{k+1} W_{2 i-2-2 k} W_{2 i_{2}} \cdots W_{2 i_{p}}\left[Y_{2} s\right]
$$

where $Y_{4 k}=\bigcup_{j=0}^{k} \mathbf{C} P^{2 k-2 j} \times N_{j}$, and $\tilde{d}$ is the direct sum of the classes $\bar{d} \in$ $H^{2}\left(\mathrm{CP}^{2^{s-1}-2 j} \times N_{j}\right)$.

The pair $\left(\mathbf{C} P^{2 k-2 j}, \gamma^{1}\right)$ can be regarded as a class in the bordism group $\Omega_{*}^{U}\left(\mathbf{C} P^{\infty}\right)[\mathbf{1}]$, where $\gamma^{1}$ is the canonical line bundle over $\mathbf{C} P^{n} . \Omega_{*}^{U}\left(\mathbf{C} P^{\infty}\right)$ has the structure of a $\Omega_{*}^{U}$ module, and so we can regard $Y_{4 k}$ as a class in $\Omega_{*}^{U}\left(\mathbf{C} P^{\infty}\right)$. There is an augmentation homomorphism $\varepsilon: \Omega_{*}^{U}\left(\mathbf{C} P^{\infty}\right) \rightarrow \Omega_{*}^{U}$ sending $(M, \xi)$ to $M$, and there is the Smith homomorphism $\Delta: \Omega_{*}^{U}\left(\mathbf{C} P^{\infty}\right) \rightarrow \Omega_{*}^{U}\left(\mathbf{C} P^{\infty}\right)$ of degree -2 sending $(M, \xi)$ to $\left(N,\left.\xi\right|_{N}\right)$, where $N$ is the submanifold of $M$ dual to $\bar{\xi}$. Both $\varepsilon$ and $\Delta$ are $\Omega_{*}^{U}$ homomorphisms, and $\Delta\left(\mathbf{C} P^{n}, \gamma^{1}\right)=\left(\mathbf{C} P^{n-1}, \gamma^{1}\right)$. In [1, (5.3)] Conner and Floyd prove that there is a unique basis $\left\{X_{2 i} \mid i=0,1,2, \ldots\right\}$ of $\Omega_{*}^{U}\left(\mathbf{C} P^{\infty}\right)$ as an $\Omega_{*}^{U}$ module such that

(i) $X_{0}=1$,

(ii) $\Delta X_{2 i}=X_{2 i-2}$ for $i>0$,

(iii) $\varepsilon X_{2 i}=0$ for $i>0$.

For $k>0$, let

$$
Y_{2 i}= \begin{cases}Y_{4 k}, & \text { if } i=2 k \\ \Delta Y_{4 k}, & \text { if } i=2 k-1 .\end{cases}
$$

Then it is easy to see that the classes $Y_{2 i}$ satisfy the three properties above, and so by uniqueness, the class of $Y_{2 i}$ in $\Omega_{*}^{U}\left(\mathbf{C} P^{\infty}\right)$ is the same as the class of $X_{2 i}$. Moreover,

$$
W_{2 i+1} W_{2 i_{2}} \cdots W_{2 i_{p}}[M]=\sum_{k=0}^{i-1} \tilde{d}^{k+1} c_{i-1-k} c_{i_{2}} \cdots c_{i_{p}}\left[X_{2^{s}}\right]
$$

where $\tilde{d}$ is the first Chern class of the line bundle over $X_{2^{s}}$ (as a class in $\Omega_{*}^{U}\left(\mathbf{C} P^{\infty}\right)$ ).

The Conner-Floyd classes $X_{2 k}$ are defined inductively. However, there is a direct construction due to Stong. Let $\xi$ be a complex $k$-plane bundle over a space $X$. Then 
$\mathbf{C P}(\xi)$ is the space of complex lines in the fibers of $\xi$. Let $\lambda$ be the complex line bundle over $\mathbf{C P}(\xi)$ whose total space consists of pairs $(\alpha, x)$, where $\alpha \in \mathbf{C P}(\xi)$ and $x$ is a point on $\alpha$. Then we can take $X_{2 k}=\left[\mathbf{C P}\left(\gamma_{1}^{1} \oplus(k-1) \mathbf{C}\right), \lambda\right]$, with a suitable complex structure, where $\gamma_{1}^{1}$ is the canonical complex line bundle over $\mathbf{C} P^{1},(k-1) \mathbf{C}$ is the trivial complex $(k-1)$-plane bundle over $\mathbf{C} P^{1}$, and $\lambda$ is the bundle of pairs as defined above. Thus, we have

$$
W_{2 i+1} W_{2 i_{2}} \cdots W_{2 i_{p}}[M]=\sum_{k=0}^{i-1} \tilde{d}^{k+1} W_{2 i-2-2 k} W_{2 i_{2}} \cdots W_{2 i_{p}}\left[\mathbf{C} P\left(\gamma_{1}^{1} \oplus\left(2^{s-1}-1\right) \mathbf{C}\right)\right]
$$

where $\tilde{d}$ is the mod 2 reduction of $c_{1}(\lambda)$. One can show $[2$, p. 36] that the total Stiefel-Whitney class of $\mathbf{C P}\left(\gamma_{1}^{1} \oplus\left(2^{s-1}-1\right) \mathbf{C}\right)$ is $W=(1+\tilde{d}+a)(1+\tilde{d})^{2^{s-1}-1}$, where $a$ is the mod 2 reduction of the first Chern class of $\mathbf{C} P^{1}$ (so $\left.a^{2}=0\right)$. Thus $W=a(1+\tilde{d})^{2^{s-1}-1}+(1+\tilde{d})^{2^{s-1}}$, and so $W_{2 j}=a \tilde{d}^{j-1}$ for $0<2 j<2^{s}$. Thus, if $p \geq 3$, then $W_{2 i+1} W_{2 i_{2}} \cdots W_{2 i_{p}}[M]=0$.

Thus, summing up we have

THEOREM 2.12. If $M$ is an n-dimensional manifold with cobcat $(M) \leq 3$, then $M$ is either a boundary or else one of the following is true:

(1) $n=2^{2}, s \geq 1$ and $M$ is cobordant to $\left(\mathbf{R} P^{2}\right)^{2^{s-1}}$.

(2) $n=2^{r}\left(2^{s}+1\right), r \geq 0, s \geq 2$ and $M$ is cobordant to $(\tilde{M})^{2^{r}}$, where

$$
\tilde{M}=\bigcup_{j=0}^{2^{s-2}} P\left(1,2^{s-1}-2 j\right) \times N_{j}
$$

and $N_{j}$ is the degree $4 j$ term in the formal inverse $\left(1+\mathrm{C} P^{2}+\mathrm{C} P^{4}+\mathrm{C} P^{6}+\cdots\right)^{-1}$.

ProOF. Suppose $M$ is not a boundary. Then by Corollary 2.9 , we may assume $\alpha(n) \leq 2$. If $\alpha(n)=1$, i.e., $n=2^{s}$, then by Corollary 2.5 , we have (1). Let $\alpha(n)=2$, i.e., $n=2^{r}\left(2^{s}+1\right)$, where $r \geq 0$ and $s \geq 1$. By Corollary 2.5, we may assume $s \geq 2$. Then, by Proposition 2.10 and Theorem 2.11, we have (2).

Since cobcat $(M) \leq \operatorname{cat}(M)$, the previous theorem also holds under the hypothesis cat $(M) \leq 3$. However the representatives given for the possible nonbounding classes above need not have $\operatorname{cat}(M)=3$, although they do satisfy cobcat $(M)=3$. In a few small dimensions the author has representatives with category three. For example in $\mathfrak{N}_{4}$ we could take $\mathbf{C} P^{2}$ instead of $\mathbf{R} P^{2} \times \mathbf{R} P^{2}$ and in $\mathfrak{N}_{8}$ we could take $\mathbf{H} P^{2}$ instead of $\left(\mathbf{R} P^{2}\right)^{4}$. The author does not know of representatives with category three for all the dimensions in (1) and (2) above, or even if they exist.

\section{REFERENCES}

1. P. E. Conner and E. E. Floyd, Torsion in SU-bordism, Mem. Amer. Math. Soc. No. 60 (1966).

2. __ Fibering within a cobordism class, Michigan Math. J. 12 (1965), 33-47.

3. A. Dold, Erzeugende der Thomschen Algebra $\mathfrak{N}$, Math. Z. 65 (1956), 25-35.

4. I. M. James, On category, in the sense of Lusternik-Schnirelmann, Topology 17 (1978), 331-348.

5. M. V. Mielke, Cobordism properties of manifolds of small category, Proc. Amer. Math. Soc. 21 (1969), 332-334.

6. J. W. Milnor, On the Stiefel-Whitney numbers of complex manifolds and spin manifolds, Topology 3 (1965), 223-230.

7. J. W. Milnor and J. D. Stasheff, Characteristic classes, Ann. of Math. Studies, No. 76, Princeton Univ. Press, Princeton, N.J., 1974. 
8. N. E. Steenrod and D. B. A. Epstein, Cohomology operations, Ann. of Math. Studies, No. 50, Princeton Univ. Press, Princeton, N.J., 1962.

9. R. E. Stong, Notes on cobordism theory, Math. Notes, Princeton Univ. Press, Princeton, N.J., 1968.

10. __ Cobordism and Stiefel-Whitney numbers, Topology 4 (1965), 241-256.

11. R. Thom, Quelques propriétés globales des variétés différentiables, Comment. Math. Helv. 28 (1954), 17-86.

12. C. T. C. Wall, Determination of the cobordism ring, Ann. of Math. 72 (1960), 292-311.

13. Wu Wen-Tsun, Les i-carrés dans une variétés grassmannienne, C.R. Acad. Sci. Paris 230 (1950), 913-920.

Department of MAThMatics, UNiversity of North Carolina at Charlotte, Charlotte, North Carolina 28223 